Check for updates

Cite this: RSC Adv., 2017, 7, 21969

Received 4th April 2017

Accepted 13th April 2017

DOI: $10.1039 / c 7 r a 03840 b$

rsc.li/rsc-advances

\section{One-step and green synthesis of nitrogen-doped carbon quantum dots for multifunctional electronics $\uparrow$}

\author{
Zhan Wang, Lijun Cao, Yamei Ding, Rui Shi, Xiangjing Wang, Hang Lu, Zhengdong Liu, \\ Fei Xiu, Juqing Liu (DD* and Wei Huang* \\ A facile one-step and green synthesis method was developed to prepare water soluble, environmentally \\ friendly, and highly nitrogen-doped carbon quantum dots (CQDs) with tunable properties. As a proof-of- \\ concept application, a multifunctional electronic device with the configuration of PEDOT:PSS/s- \\ CQDASWCNT/PEDOT:PSS was fabricated through a full-solution process, which exhibits memory and \\ current limiting behavior, with the merit of stable operation and reliable endurance.
}

Carbon electronic materials, including carbon quantum dots (CQDs), fullerenes, carbon nanotubes (CNTs), graphene, and graphdiyne, have aroused extensive attention due to their novel structures, and unique electrical and optical properties. ${ }^{1-4}$ Among them, CQDs have attained a prominent position in the field of nanoscience and nanotechnology owing to their large specific surface area, low toxicity, excellent solubility and highly tunable properties. ${ }^{5-7}$ Several attempts, such as laser irradiation, ${ }^{8}$ microwave irradiation, ${ }^{9}$ electrochemical, ${ }^{10}$ chemical cutting, ${ }^{11}$ and hydrothermal routes ${ }^{12}$ have been reported to realize the controllable synthesis of CQDs with tunable properties. So far, compared with other mentioned methods, hydrothermal approach is one of the most efficient and effective methods to prepare CQDs, with the merit of simple process, large-scale production, and potential low cost. The properties of CQDs can be tuned via doping or surface functionalization, ${ }^{13-20}$ but some of the most common challenges faced by multi-step synthesis, expensive carbon sources, low yield, complex preparation and post-treatment associating with toxic chemicals. Utilization of one-step synthesis, nontoxic chemicals, environmentally benign solvents, and renewable source materials are highly desirable in the synthesis of doped CQDs.

Because of their outstanding physical and chemical properties, CQDs and their derivatives, especially for doped CQDs, have been used successfully in the field of bioimaging, catalysis and sensor issues. ${ }^{21-23}$ Recently, doped and undoped CQDs also show promising applications in the next generation of organic electronic and optoelectronic devices, such as solar cells for

Key Laboratory of Flexible Electronics (KLOFE) \& Institute of Advanced Materials (IAM), Jiangsu National Synergistic Innovation Center for Advanced Materials (SICAM), Nanjing Tech University (NanjingTech), 30 South Puzhu Road, Nanjing 211816, P. R. China.E-mail: iamjqliu@njtech.edu.cn; iamwhuang@njtech.edu.cn

$\dagger$ Electronic supplementary information (ESI) available. See DOI: 10.1039/c7ra03840b energy conversion, light-emitting devices for display and lighting, memories for data storage, and photodetectors for information collection. ${ }^{24-27}$ However, most of the CQDs-based organic electronics focus on the actualization of single function in a single device, but there has been a lack of breakthroughs in the multifunctional electronic devices. Therefore, how to realize CQDs-based multifunctional devices is extremely important.

In this work, we present a facile one-step and green approach for the synthesis of nitrogen-doped CQDs via a simple hydrothermal method. Our strategy is renewable, inexpensive, nontoxic, and environmentally friendly since sustainable vegetables and deionized water are the choice of self-doped carbon sources and solvent medium, respectively. Importantly, this method is a general process and can be used to prepare various nitrogen-doped CQDs from different precursors. As a proof of concept, the hybrid nitrogen-doped CQDs and single-walled carbon nanotubes (SWCNTs) film is employed as active materials in carbon electronics with PEDOT:PSS film as both of the electrodes, the solution processable device does not exhibit volatile memory effect, but also features a current limiting behavior.

Self-doped carbon sources, including spinach, Guangdong cabbage, and brassicachinensis 1 , were purchased from local markets as precursor. In order to synthesize CQDs, $100 \mathrm{~g}$ precursor and $20 \mathrm{~mL}$ deionized water were squeezed and added into a $50 \mathrm{~mL}$ Teflon-lined stainless steel autoclave, then maintained at $180^{\circ} \mathrm{C}$ for $12 \mathrm{~h}$. After the reaction and cooling process, dark brown solution was filtered with a $0.22 \mu \mathrm{m}$ membrane followed by dialyzing using $1 \mathrm{kDa}$ dialysis membrane for 1 day to remove the large particles. Finally, CQDs were collected from the light yellow aqueous solution and stored at $4{ }^{\circ} \mathrm{C}$ for further characterization and device fabrication.

Fig. 1a shows the transmission electron microscopy (TEM) image, which displays that the spinach-CQDs (referred as s- 

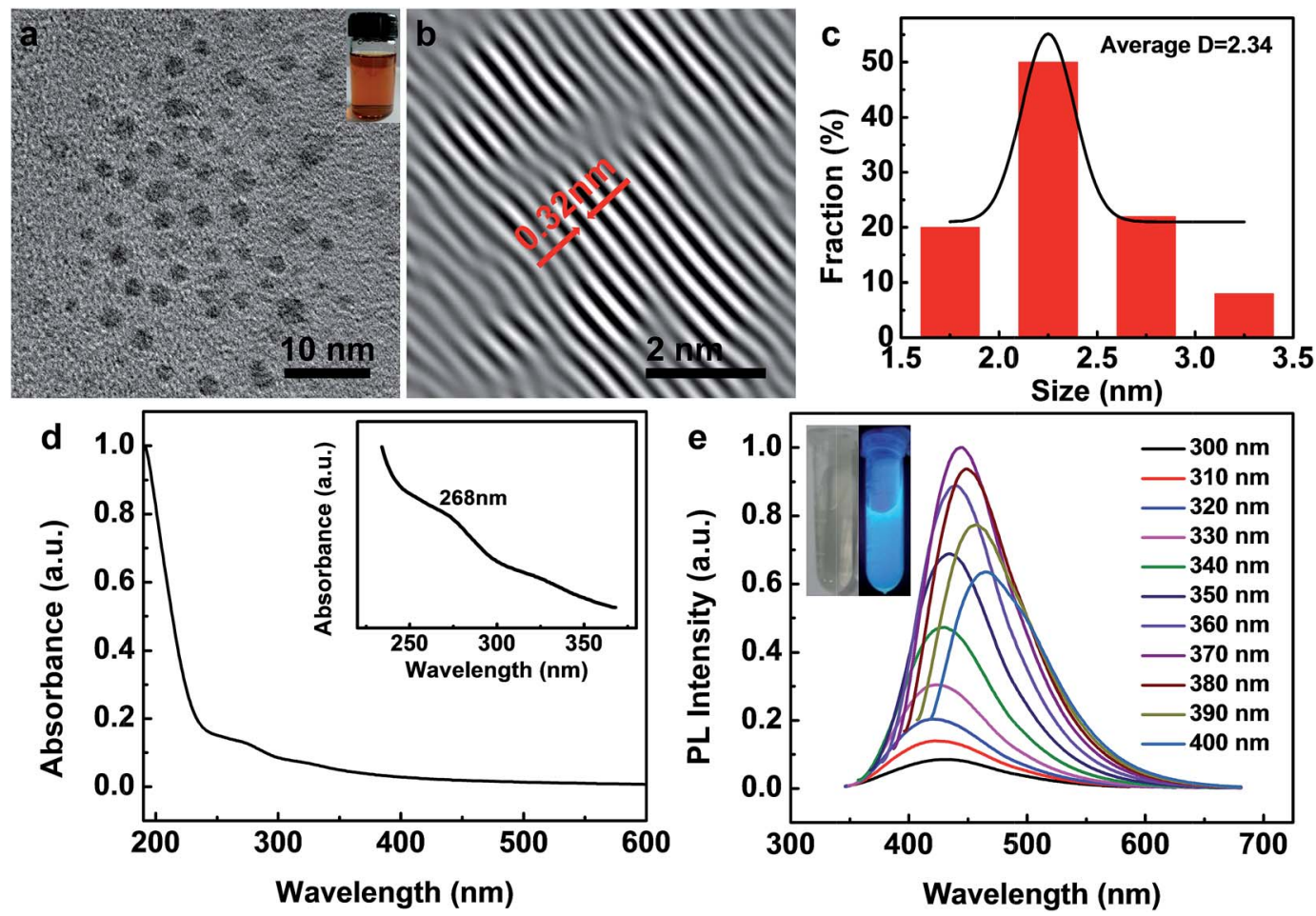

Fig. 1 (a) TEM images of as-prepared s-CQDs. (b) Inverse fast Fourier transform (IFFT) algorithm of s-CQDs. Inset: photograph of the concentration s-CQD aqueous solution. (c) Size distribution of s-CQDs. (d) UV-visible absorption and (e) photoluminescence spectra of s-CQDs in water, respectively; inset in (e) is a photo of dilute s-CQD aqueous solution under visible and UV irradiation (365 nm), respectively.

CQDs) are spherical particles well dispersed in water with a mean diameter of $2.34 \mathrm{~nm}$ (Fig. 1c), much smaller than that (ca. $10 \mathrm{~nm}$ ) of graphene quantum dots (GQDs) prepared by other hydrothermal methods. ${ }^{28}$ In the inverse fast Fourier transform (IFFT) algorithm image (Fig. 1b), the s-CQDs feature a kind of lattice fringe, with an in-plane lattice spacing of $0.32 \mathrm{~nm}$. The value of lattice spacing is very close to that of graphite $(0.34 \mathrm{~nm})$, indicating a graphite structure in the crystalline core of s-CQDs. ${ }^{29}$

To investigate the optical properties, UV-visible absorption spectrum of the as-synthesized s-CQDs in water were carried out. Fig. 1d shows a weak absorption band at $c a .268 \mathrm{~nm}$, which is attributed to the $\pi-\pi^{*}$ electron transition of $\mathrm{C}=\mathrm{C}$ bond, similar to CQDs obtained by the carbonization of cellulosebased materials. ${ }^{30}$ The photoluminescent (PL) spectrum shows a strong emission peak at $430 \mathrm{~nm}$ when excited at $c a .268 \mathrm{~nm}$ (Fig. 1e). Meanwhile, the emission spectra of as-prepared sCQDs also show an excitation-dependent feature, very similar to that of microwave synthesized C-dots. ${ }^{31}$ Inset of Fig. 1e shows the s-CQDs aqueous solution under visible and UV irradiation at $365 \mathrm{~nm}$, the bright blue PL of s-CQDs under UV light is obviously observed. Moreover, the full width at half-maximum is $86 \mathrm{~nm}$, indicating a relatively small size distribution of dots, which is consistent with the TEM data and previous reported values. ${ }^{32}$

Fig. 2a shows typical XRD profiles for as-prepared s-CQDs. From XRD patterns, a broad peak centered at around $20.92^{\circ}$ could be found, which is similar to a graphite lattice spacing $\left(20.73^{\circ}\right) .^{33}$ This lattice contraction in the above range is common for nanomaterials in nanoscale regime, which is probably attributed to rich active sites on the surface of s-CQDs induced by the hydrothermal process. ${ }^{10}$ Furthermore, XRD results also confirm the lattice spacing of $3.2 \AA$. Meanwhile, Raman spectra have been performed to explore the structure of s-CQDs (Fig. 2b). The major Raman features are the $\mathrm{D}$ band at $c a$. $1365 \mathrm{~cm}^{-1}$ and the G band at $c a .1566 \mathrm{~cm}^{-1}$. Unlike the GQDs synthesized electrochemically with a much less intense D band, ${ }^{10}$ the relative intensity of the disorder D-band and crystalline G-band $\left(I_{\mathrm{D}} / I_{\mathrm{G}}\right)$ for s-CQDs in this work is approximately 0.75 and much smaller than the value (0.84) of GQDs synthesized by microwave method. The above findings not only indicate our s-CQDs exhibiting lower defects and higher quality, but also demonstrate the uniqueness of this simple and green synthesis method. ${ }^{34}$

X-ray photoelectron spectroscopy (XPS) measurements were performed to probe the chemical composition of s-CQDs. As
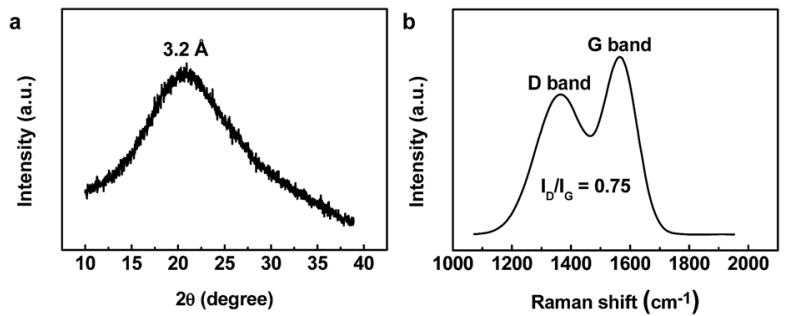

Fig. 2 (a) XRD patterns and (b) Raman spectra of as-prepared s-CQDs. 
shown in Fig. 3a, the XPS survey scans display three dominant peaks at 284.6, 399.0, and $532.0 \mathrm{eV}$, corresponding to $\mathrm{C} 1 \mathrm{~s}, \mathrm{~N} 1 \mathrm{~s}$, and $\mathrm{O} 1 \mathrm{~s}$, respectively. ${ }^{35}$ The strong nitrogen signal indicates the successful nitrogen-doping of s-CQDs owing to the abundant nitrogen elements in vegetables. The surface atomic concentration of carbon, nitrogen, and oxygen is $66.39 \%, 7.73 \%$, and $27.88 \%$, respectively. The four deconvoluted peaks in the high resolution $\mathrm{C}$ 1s spectrum (Fig. 3b) suggest the presence of four types of carbon bonds, i.e., $\mathrm{C}=\mathrm{C}$ at $284.1 \mathrm{eV}, \mathrm{C}-\mathrm{C}$ at $284.9 \mathrm{eV}$, $\mathrm{C}-\mathrm{N}$ at $285.9 \mathrm{eV}$, and $\mathrm{C}-\mathrm{O}-\mathrm{C}=\mathrm{O}$ at $287.3 \mathrm{eV} \cdot{ }^{36-39}$ Fig. $3 \mathrm{c}$ shows the high-resolution spectrum of $\mathrm{O} 1 \mathrm{~s}$, from which three kinds of carbon-oxygen bond including $\mathrm{C}-\mathrm{O}, \mathrm{C}=\mathrm{O}$ and $\mathrm{O}-\mathrm{C}=\mathrm{O}$ could be found, corresponding to the peak at 530.5, 531.6 and $532.7 \mathrm{eV}$, respectively. ${ }^{31,40}$ The high-resolution $\mathrm{O}$ 1s peak indicates that oxidation reaction took place during the hydrothermal treatment. In the high-resolution $\mathrm{N}$ 1s spectrum, two peaks at 398.4 and $399.7 \mathrm{eV}$ are observed in terms of pyridinelike $\mathrm{N}$ and pyrrolie-like $\mathrm{N}$ species, respectively. ${ }^{35,41}$ These findings suggest that $\mathrm{N}$-doped $\mathrm{s}$-CQDs are decorated by abundant hydroxyl and carboxyl groups, which is in consistent with the results shown in FT-IR spectrum (Fig. S3†). Therefore, s-CQDs could be stable and well dispersed in water due to the existence of those functional groups.

More importantly, we also successfully synthesized other types of CQDs based on Guangdong cabbage (referred as $g$ CQDs) and brassicachinensis 1 (referred as $b$-CQDs) carbon sources. Fig. S1 $\uparrow$ shows the TEM images of $g$-CQDs and $b$-CQDs, which give similar morphology with that of s-CQDs. The average sizes of $g$-CQDs and $b$-CQDs is approximately $2.06 \mathrm{~nm}$ and $3.31 \mathrm{~nm}$, respectively, which is comparable with the value (2.34 $\mathrm{nm}$ ) of s-CQDs. Fig. S2 $\uparrow$ displays the UV-visible absorption and PL spectra, from which a weak absorption peak and strong emission peak at $272 \mathrm{~nm}$ and $418 \mathrm{~nm}$ for $g$-CQDs, $268 \mathrm{~nm}$ and $435 \mathrm{~nm}$ for $b$-CQDs could be observed, which is slightly different from the value $(268 \mathrm{~nm}, 430 \mathrm{~nm})$ of s-CQDs. Above results demonstrate that our method can offer a facile and universal green synthesis approach for the preparation of various CQDs. Other details such as their particle structure, chemical
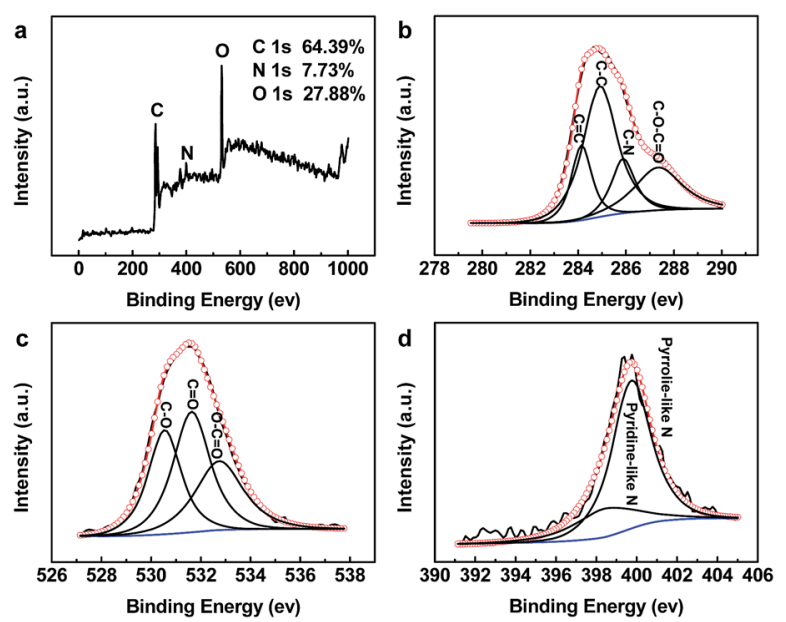

Fig. 3 (a) XPS spectra of s-CQDs. (b) C 1s, (c) $O 1$ s, and (d) N 1s spectra analysis of the as-prepared s-CQDs. composition, electrical and optical properties of these CQDs are under investigation.

Since the s-CQDs are green carbon semiconductors, the hybrid S-CQD and SWCNT composite (referred as sCQD@SWCNT) is a promising electroactive material for electronic devices. As a proof-of-concept application, the properties of s-CQD@SWCNT and its application in diode is studied. A typical in-planar diode with the configuration of poly(3,4ethylenedioxythiophene):polystyrene sulfonate (PEDOT:PSS)/sCQD@SWCNT/PEDOT:PSS was fabricated (Fig. 4a), where s-CQD@SWCNT acted as the electroactive layer. It should be noted that hysteresis is a critical factor to evaluate a memory effect and an obvious hysteresis behaviour is observed when the voltage is swept from -5 to $5 \mathrm{~V}$ and back to $-5 \mathrm{~V}$ (Fig. $4 \mathrm{~b}$ ). The current increase progressively when the voltage increases from 0 to $1 \mathrm{~V}$ (stage I) or decreases from 0 to $-2 \mathrm{~V}$ (stage III), and pronounced negative differential resistance (NDR) occurs at the voltage of $c a .+1 \mathrm{~V}$ in stage I (or $-2 \mathrm{~V}$ in stage III). NDR can normally be attributed to the charge trapping of nanomaterials. ${ }^{42}$ With the decrease of voltage (stages II and IV), a hysteresis phenomenon is observed, indicating an electrical transition from low resistance state (ON) to high resistance state (OFF). This transition is equivalent to a set/write process in digital memory. Note that the memory state cannot be stored when the power is switched off, suggesting its volatile nature. Compared to nonvolatile flash and write-once-read-many-times (WORM) memories, ${ }^{43-45}$ the fabricated device exhibits a dynamic random access memory (DRAM), which is also different from the previous reports on the devices with either CNT or isolated CQD as the electroactive layer, ${ }^{46,47}$ implying that the observed phenomenon is an intrinsic property of the hybrids. Moreover, this memory device exhibits repeated hysteresis loops without any significant degradation, promising excellent stability during the device operation. It's worth pointing out that there has been much work in functionalized CNT devices that also exhibit stability. ${ }^{\mathbf{4 8 , 4 9}}$

Because of the pronounced NDR features, the current of hybrid device increases firstly and subsequent decreases slowly
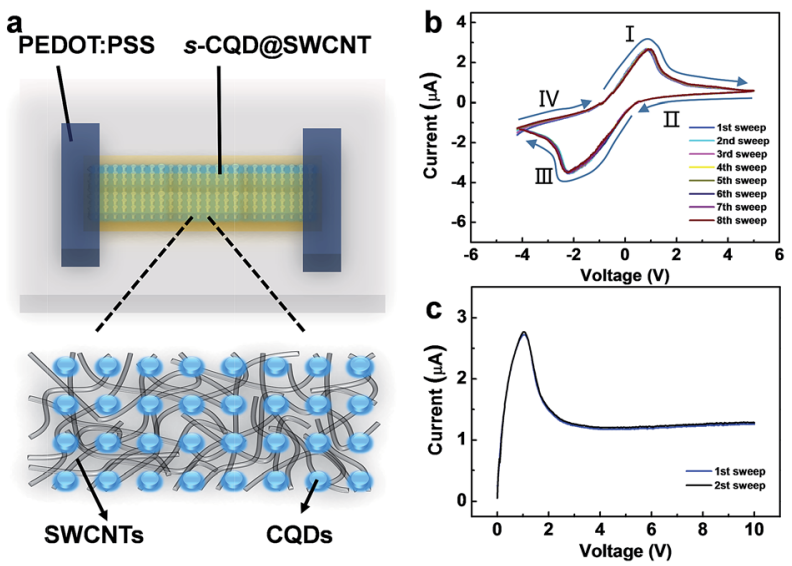

Fig. 4 (a) Schematic drawing illustrating the configuration of a multifunctional device with s-CQDaSWCNT as the electroactive layer. (b) and (c) $I-V$ characteristics for the multifunctional device at a scan rate of $20 \mathrm{mV} \mathrm{s}^{-1}$. 
with the increase of voltage to a constant value $(\sim \mu \mathrm{A})$, meaning the device is capable of imposing an upper limit on the current. The current-limiting behaviour may be used to protect the circuit generating or transmitting the current from harmful effects. One of the most impressive features is that this hybrid device shows outstanding endurance ability and no degradation is observed in the $I-V$ curves during the recycle testing (Fig. $4 \mathrm{c}$ ). Therefore, the as-fabricated device not only exhibits memory behaviour, but also features current-limiting ability. It is worth pointing out that the role of s-CQD is very important in achieving multifunctional properties of device. Our control experiments with isolated SWCNTs as electroactive layer show no evidence of hysteresis and NDR phenomenon, indicating that the memory and current-limiting effect is attributed to the existence of s-CQD in the hybrid nanostructures.

In summary, we present a facile one-step approach to produce water soluble, highly N-doped CQDs through a completely green synthesis method. This combination of environmentally friendly reactants and solvent present a wide range of possibility for the synthesis of green nitrogen-doped carbon electronic materials. As a proof-of-concept application, the hybrid N-doped CQD and SWCNT film was successfully used as electroactive layer in multifunctional electronic devices with a configuration of PEDOT:PSS/s-CQD@SWCNT/PEDOT:PSS. The fabricated device exhibits volatile memory and current limiting behaviour, with the merit of stable operation and reliable endurance. Importantly, the full-solution process fabrication technology make this device extremely simple and potential low cost. We believe that this study not only provides a new path to design multifunctional carbon electronics constructed from hybrid nanostructures, but also boosts the development of carbon electronics and green electronics.

\section{Acknowledgements}

The authors gratefully acknowledge the financial support from National Key Basic Research Program of China (973 Program, 2015CB932200), the National Natural Science Funds for Excellent Young Scholars (61622402), the National Natural Science Foundation of China (61376088, 51302134, 51528201), the Natural Science Foundation of Jiangsu Province (BK20150955), the Jiangsu Specially-Appointed Professor programme, and the Six Talent Plan (2015XCL015).

\section{Notes and references}

1 H. T. Li, X. D. He, Z. H. Kang, H. Huang, Y. Liu, J. L. Liu, S. Y. Lian, C. H. A. Tsang, X. B. Yang and S. T. Lee, Angew. Chem., Int. Ed., 2010, 49, 4430.

2 P. Avouris, M. Freitag and V. Perebeinos, Nat. Photonics, 2008, 2, 341.

3 K. S. Novoselov, A. K. Geim, S. V. Morozov, D. Jiang, Y. Zhang, S. V. Dubonos, V. Grigorieva and A. A. Firsov, Science, 2004, 306, 666.

4 G. Z. Sun, J. Q. Liu, L. X. Zheng, W. Huang and H. Zhang, Angew. Chem., Int. Ed., 2013, 52, 13351.
5 S. Kim, S. W. Hwang, M. K. Kim, D. Y. Shin, C. O. Kim, S. B. Yang, J. H. Park, E. Hwang, S. H. Choi, G. W. Ko, S. H. Sim, C. S. Sone, H. J. CHoi, S. K. Bae and B. H. Hong, ACS Nano, 2012, 6, 8203.

6 A. B. Bourlinos, A. Stassinopoulos, D. Anglos, R. Zboril, M. Karakassides and E. P. Giannelis, Small, 2008, 4, 455.

7 L. Wang, S. J. Zhu, H. Y. Wang, S. N. Qu, Y. L. Zhang, Q. D. Chen, H. L. Xu, W. Han, B. Yang and H. B. Sun, ACS Nano, 2014, 8, 2541.

8 L. A. Ponomarenko, F. Schedin, M. I. Katsnelson, R. Yang, E. W. Hill, K. S. Novoselov and A. K. Geim, Science, 2008, 320, 356.

9 L. L. Li, J. Ji, R. Fei, C. Z. Wang, Q. Lu, J. R. Zhang, L. P. Jing and J. J. Zhu, Adv. Funct. Mater., 2012, 22, 2971.

10 Y. Li, Y. Hu, Y. Zhao, G. Q. Shi, L. E. Deng, Y. B. Hou and L. T. Qu, Adv. Mater., 2011, 23, 776.

11 Y. Q. Sun, S. Q. Wang, C. Li, P. H. Luo, L. Tao, Y. Wei and G. Q. Shi, Phys. Chem. Chem. Phys., 2013, 15, 9907.

12 B. D. Pan, J. Zhang, Z. Li and M. Wu, Adv. Mater., 2010, 22, 734.

13 Y. H. Yang, J. H. Cui, M. T. Zheng, C. F. Hu, S. Z. Tan, Y. Xiao, Q. Yang and Y. L. Liu, Chem. Commun., 2012, 48, 380.

14 P. Ayala, R. Arenal, A. Loiseau, A. Rubio and T. Pichler, Rev. Mod. Phys., 2010, 82, 1843.

15 M. Zheng, S. Liu, J. Li, D. Qu, H. F. Zhao, X. G. Guan, X. L. Hu, Z. G. Xie, X. B. Jing and Z. C. Sun, Adv. Mater., 2014, 26, 3554.

16 Y. Q. Dong, R. X. Wang, H. Li, J. W. Shao, Y. W. Chi, X. M. Lin and G. N. Chen, Carbon, 2012, 50, 2810.

17 H. X. Zhao, L. Q. Liu, Z. De Liu, Y. Wang, X. J. Zhao and C. Z. Huang, Chem. Commun., 2011, 47, 2604.

18 H. L. Li, Y. W. Zhang, L. Wang, J. Q. Tian and X. P. Sun, Chem. Commun., 2011, 47, 961.

19 F. Wang, Z. Xie, H. Zhang, C. Y. Liu and Y. G. Zhang, Adv. Funct. Mater., 2011, 21, 1027.

20 Y. Mao, Y. Bao, D. X. Han, F. H. Li and L. Niu, Biosens. Bioelectron., 2012, 38, 55.

21 S. N. Baker and G. A. Baker, Angew. Chem., Int. Ed., 2010, 49, 6726.

22 R. H. Liu, H. Huang, H. T. Li, Y. Liu, J. Zhong, Y. Y. Li, S. Zhang and Z. H. Kang, ACS Catal., 2014, 4, 328.

23 S. Liu, J. Q. Tian, L. Wang, Y. W. Zhang, X. Y. Qin, Y. L. Luo, A. M. Asiri, A. O. Al-Youbi and X. P. Sun, Adv. Mater., 2012, 24, 2037.

24 Y. Q. Zhang, D. K. Ma, Y. G. Zhang, W. Chen and S. M. Huang, Nano Energy, 2013, 2, 545.

25 X. Zhang, Y. Zhang, Y. Wang, S. Kalytchuk, S. V. Kershaw, Y. Wang, P. Wang, T. Zhang, Y. Zhao and H. Zhang, ACS Nano, 2013, 7, 11234.

26 Y. R. Kim, Y. E. Jo, Y. S. Shin, W. T. Kang, Y. H. Sung, U. Y. Won, Y. H. Lee and W. J. Yu, Appl. Phys. Lett., 2015, 106, 103105.

27 S. H. Cheng, T. M. Weng, M. L. Lu, W. C. Tan, J. Y. Chen and Y. F. Chen, Sci. Rep., 2013, 3, 2694.

28 D. Y. Pan, J. C. Zhang, Z. Li and M. H. Wu, Adv. Mater., 2010, 22, 734 .

29 S. Iijima, Nature, 1991, 354, 56. 
30 J. Peng, W. Gao, B. K. Gupta, Z. Liu, R. Romero-Aburto, L. Ge, L. Song, L. B. Alemany, X. Zhan, G. Gao, S. A. Vithayathil, B. A. Kaipparettu, A. Marti, T. Hayashi, J. J. Zhu and P. Ajayan, Nano Lett., 2012, 12, 844.

31 J. Wang, F. Peng, Y. M. Lu, Y. L. Zhong, S. Y. Wang, M. F. Xu, X. Y. Ji, Y. Y. Su, L. S. Liao and Y. He, Adv. Opt. Mater., 2014, 3, 103.

32 Y. L. Hao, Z. X. Gan, X. B. Zhu, T. H. Li, X. L. Wu and P. K. Chu, J. Phys. Chem. C, 2015, 119, 2956.

33 W. Kratschmer, L. Lamb, L. Fostiropoulos and D. Huffman, Nature, 1990, 347, 354.

34 Z. M. Luo, G. Q. Qi, K. Y. Chen, M. Zou, L. H. Yuwen, X. W. Zhang, W. Huang and L. H. Wang, Adv. Funct. Mater., 2016, 26, 2739.

35 W. Ai, Z. M. Luo, J. Jiang, J. H. Zhu, Z. Z. Du, Z. X. Fan, L. H. Xie, H. Zhang, W. Huang and T. Yu, Adv. Mater., 2014, 26, 6186.

36 S. Sahu, B. Behera, T. K. Maiti and S. Mohapatra, Chem. Commun., 2012, 48, 8835.

37 G. X. Chen, Z. W. Zhuo, K. Ni, N. Y. Kim, Y. Zhao, Z. W. Chen, B. Xiang, L. H. Yang, Q. Zhang, Z. Lee, X. J. Wu, R. S. Ruoff and Y. W. Zhu, Small, 2015, 11, 5296.

38 K. Jiang, S. Sun, L. Zhang, Y. Lu, A. G. Wu, C. Z. Cai and H. W. Lin, Angew. Chem., Int. Ed., 2015, 54, 5360.
39 S. Y. Park, H. U. Lee, E. S. Park, S. C. Lee, J. W. Lee, S. W. Jeong, C. H. Kim, Y. C. Lee, Y. S. Huh and J. Lee, ACS Appl. Mater. Interfaces, 2014, 6, 3365.

40 A. Prasannan and T. Imae, Ind. Eng. Chem. Res., 2013, 52, 15673.

41 J. Tang, J. Liu, C. L. Li, Y. Q. Li, M. O. Tade, S. Dai and Y. Yamauchi, Angew. Chem., Int. Ed., 2015, 54, 588.

42 R. J. Tseng, J. Ouyang, C. W. Chu, J. S. Huang and Y. Yang, Appl. Phys. Lett., 2006, 88, 123506.

43 J. Q. Liu, Z. Y. Zeng, X. H. Cao, G. Lu, L. H. Wang, Q. L. Fan, W. Huang and H. Zhang, Small, 2012, 8, 3517.

44 J. Q. Liu, Z. Y. Yin, X. H. Cao, F. Zhao, L. H. Wang, W. Huang and H. Zhang, Adv. Mater., 2013, 25, 233.

45 J. Q. Liu, Z. Y. Yin, X. H. Cao, F. Zhao, A. P. Lin, L. H. Xie, Q. L. Fan, F. Boey, H. Zhang and W. Huang, ACS Nano, 2010, 4, 3987.

46 A. D. Liao, P. T. Araujo, R. J. Xu and M. S. Dresselhaus, Nat. Commun., 2014, 5, 5673.

47 Z. Ma, Y. L. Zhang, L. Wang, H. Ming, H. T. Li, X. Zhang, F. Wang, Y. Liu, Z. H. Kang and S. T. Lee, ACS Appl. Mater. Interfaces, 2013, 5, 5080.

48 A. C. Ford, M. Shaughnessy, B. M. Wong, A. A. Kane, O. V. Kuznetsov, K. L. Krafcik, W. E. Billups, R. H. Hauge and F. Leonard, Nanotechnology, 2013, 24, 105202.

49 X. J. Zhou, T. Zifer, B. M. Wong, K. L. Krafcik, F. Léonard and A. L. Vance, Nano Lett., 2009, 9, 1028. 